\title{
The Dynamic Game of Knowledge Hiding Behavior from Organizational Members: To Hide or Not to Hide?
}

\author{
Fan Yang $\left(\mathbb{D},{ }^{1,2}\right.$ Long Yang $\left(\mathbb{D},{ }^{3}\right.$ and Ping $\mathrm{Li} \mathbb{D}^{1}$ \\ ${ }^{1}$ College of Management Science, Chengdu University of Technology, Chengdu 610059, China \\ ${ }^{2}$ Social Science Department, Chengdu University, Chengdu 610106, China \\ ${ }^{3}$ Business School, Chengdu University, Chengdu 610106, China \\ Correspondence should be addressed to Ping Li; 513022a2cxe.cdb@sina.cn
}

Received 2 May 2021; Revised 13 July 2021; Accepted 7 August 2021; Published 6 September 2021

Academic Editor: Yi Su

Copyright (C) 2021 Fan Yang et al. This is an open access article distributed under the Creative Commons Attribution License, which permits unrestricted use, distribution, and reproduction in any medium, provided the original work is properly cited.

\begin{abstract}
In the era of the knowledge economy, it is urgent for organizations to solve the problem of knowledge hiding of internal members to accelerate the speed and efficiency of knowledge dissemination and innovation and adapt to rapid changes in the market. At present, research on knowledge hiding has received extensive attention from Western countries, but there are few relevant studies in China. Based on the hypothesis of bounded rationality, this paper constructs an evolutionary game model of second-level knowledge hiding of organizational members and analyzes the main factors affecting the stable equilibrium point using MATLAB numerical simulation. The results show that knowledge leakage risk is positively correlated with knowledge hiding. The ability of knowledge absorption and transformation is positively correlated with the behavior of knowledge hiding. There is a negative correlation between collaborative innovation ability and knowledge hiding. There is a negative correlation between knowledge stock and knowledge hiding. Only when the incentive reaches a certain level can organizational members be encouraged to give up knowledge hiding. This paper provides a more comprehensive and dynamic picture of the evolutionary game of knowledge hiding among members in the organization and provides a new idea of knowledge management for organizational managers.
\end{abstract}

\section{Introduction}

With the emergence of knowledge-based view theory and the development of strategic management theory, knowledge is widely regarded as an important resource for organizational development [1]. Knowledge-based view theory focuses on knowledge as the driver of strategy and firm performance. Therefore, organizational knowledge is a critical strategic resource for organizational success and performance improvements [2-4]. In addition, knowledge sharing is beneficial for realizing knowledge innovation [5-7] and promoting organizational performance output [8-11]. Adequate knowledge sharing needs good knowledge management system support. A successful knowledge management system depends on the behavior of organizational members, especially the behavior of knowledge sharing among organizational members [12]. However, for all the investment that managers have made in promoting knowledge sharing, the reality has not been as smooth as it might have been. Organizations are not the owners of individual knowledge assets, and individuals have no obligation to transfer their unique knowledge resources to other organizational members. Knowledge hiding behavior is common in organizations [13-15]. A statistical study of knowledge workers in China found that $38.8 \%$ of them exhibited knowledge hiding behaviors, such as being unwilling to transfer knowledge to colleagues and refusing to provide useful information for colleagues [16]. It is estimated that the top 500 companies worldwide lose more than $\$ 31.5$ billion each year due to the failure of employees to share knowledge effectively [13]. Knowledge hiding is the deliberate hiding of individuals in an organization when faced with knowledge requests, which will inhibit innovation and weaken organizational performance [17]. Moreover, it will harm the interpersonal relationships between the members of the organization and affect the creativity of the members [18]. For organizations, it is complicated system engineering to understand the complex causes of knowledge hiding and 
to intervene in the negative effects of irrational knowledge hiding behaviors of organizational members. However, for a long time, knowledge hiding has not received enough attention from theoretical circles. In recent years, it has attracted wide attention from scholars and developed into a frontier topic of organizational behavior research [19]. Based on the perspectives of organizational management, leadership management, and individual characteristics, the theoretical circle has summarized the influencing factors of knowledge hiding using inductive and multicase analyses and tested the influencing mechanism of knowledge hiding using regression analysis and a structural equation model. However, there is a lack of systematic analysis of different influencing factors from multiple perspectives. In addition, the knowledge hiding of organizational members changes dynamically with changes in the organizational environment and leadership behavior; thus, it is difficult to scientifically and comprehensively reflect the process of the generation and change of knowledge hiding among organizational members only by analyzing knowledge hiding from a static perspective. Therefore, this paper intends to build an evolutionary game model of organizational member knowledge hiding, analyze the dynamic changes and stabilization strategies of organizational member knowledge hiding from a multidimensional perspective with different parameter variables, and use numerical simulation to reflect the influence of dynamic changes in different parameters on knowledge hiding strategies. On the one hand, the research results can provide a reference for the research methods of knowledge hiding and expand knowledge management theory. On the other hand, it can provide a useful practical reference for managers to prevent the occurrence of knowledge hiding of organizational members in advance and break the vicious circle of knowledge hiding afterwards.

\section{Theoretical Background and Literature Review}

According to the cognitive-motivational-relational theory of emotion $[20,21]$, people are motivated to react if they appraise an environment, or encounter, as personally meaningful. An event that hinders or facilitates an individual's goal may be appraised as either a harm, a threat, a challenge, or a benefit. Hence, if the knowledge request is regarded as harmful or threatening, knowledge hiding may occur. The urgent problem to be solved in the process of knowledge management is to create an organizational environment that stimulates knowledge sharing and innovation and reduce the knowledge hiding of organizational members [22]. To gain a deep understanding of the research status in the field of knowledge hiding of organizational members, "knowledge hiding" was searched as the topic in the Web of Science database and the China National Knowledge Infrastructure database. The highly cited literature and hot literature related to our research in the Web of Science database were read. The source journals of the Chinese Social Sciences Citation Index relying on our research in the China National Knowledge Infrastructure database were read. Existing studies have defined the concept of knowledge hiding [14] and clarified the causes and consequences of knowledge hiding from different perspectives [15].

2.1. The Concept of Knowledge Hiding. Connely et al. [14] defined knowledge hiding as the behavior in which a member intentionally retains or hides knowledge when others in an organization put forward a demand for knowledge. Knowledge hiding is divided into three categories: deceptive knowledge hiding, evasive knowledge hiding, and rationalized knowledge hiding. Deceptive knowledge hiding refers to deliberately playing dumb when asked. Evasive knowledge hiding refers to providing only partial knowledge or even misleading knowledge when asked. Rationalized knowledge hiding is refusing to provide knowledge for reasonable reasons. Knowledge hiding is not always a malicious act, but it can often hinder the learning development of individuals, teams, and organizations [14]. Jha and Varkkey [23] proposed a new type of counterquestioning knowledge hiding, which is reflected when one asks questions, and a question is raised by the other party in the form of a rhetorical question. And Yuan et al. [24] proposed another type of bullying hiding based on the knowledge hiding classification proposed by Connely. These definitions enrich the classification of knowledge hiding. Connelly and Zweig [25] studied the effects of different types of knowledge hiding on colleague relationships. Rationalized knowledge hiding would not harm the relationships among members but would strengthen the relationships among colleagues and even break the cycle of knowledge hiding in the organization. Evasive knowledge hiding and deceptive knowledge hiding can hurt relationships between members in an organization. Specifically, deceptive knowledge hiding does not lead to others' knowledge hiding in the future; only evasive knowledge hiding will lead to this hiding. Because evasive knowledge hiding includes knowledge sharing, it is not suitable to be analyzed as pure knowledge hiding. Connelly and Zweig [25] found that rational knowledge hiding does not damage the relationship between organizational members. Therefore, knowledge hiding in this study refers to deceptive knowledge hiding.

\subsection{Organizational Management and Knowledge Hiding.} Connelly and Kelloway [26] used regression analysis to prove that management support and a positive culture of social interaction are conducive to promoting knowledge sharing among employees. Abubakar et al. [27] applied artificial neural networks to prove that the fairer organizational management, the lower the degree of knowledge hiding. Through regression analysis, Ghani et al. [28] found that abusive management would destroy the psychological contract of organizational members, which would lead to knowledge hiding. Jiang et al. [29] used regression analysis to verify that the higher the degree of organizational cynicism, the greater the negative impact of knowledge hiding on employees' psychological security, and the greater the negative impact on employees' personal development. Zhu et al. [30] used regression analysis to prove that individual- 
centered performance feedback will produce knowledge hiding behavior, while team-centered performance feedback will not. Su and $\mathrm{Yu}$ [31] pointed out that when individual patents are excessively concentrated, the dissemination and sharing of knowledge will be seriously affected. It can be seen that humanized management and team-oriented incentive mechanisms are conducive to breaking the knowledge hiding among members of the organization. From the perspective of human resource management practice, Černe et al. [32] applied regression analysis to prove that when organizational members produce knowledge hiding behaviors, if accompanied by the working characteristics of high task-dependent or high decision-making autonomy, the organizational atmosphere will promote the generation of high-level innovative work behaviors. Fong et al. [33] used regression analysis to verify that task-dependent job design can weaken the negative correlation between knowledge hiding and absorptive capacity and enhance team creativity. Gagné et al. [34] proved through the ESEM model that job cognition needs and work autonomy have a positive and significant impact on knowledge sharing frequency. In addition, Singh [10] used an SEM model to test whether job territoriality may also result in a certain degree of knowledge hiding and affect work performance. Zhao et al. [35] used the HLM model to further prove that exclusion in the workplace is positively correlated with evasive knowledge hiding and deceptive knowledge hiding, and the higher the degree of negative reciprocity belief and moral detachment, the more significant the relationship. Su et al. [36] confirmed in a study on the characteristics of knowledge flows among subjects in a regional innovation system that when the relationship between the subjects of the regional innovation system reaches a certain strength, the knowledge flow in the system exhibits the emergence of a fast flow rate. Scientific job design can promote knowledge transfer and absorption among organizational members and achieve the innovation performance of individuals, teams, and organizations. Job territoriality and exclusion in the workplace will lead to knowledge hiding.

2.3. Leaders and Knowledge Hiding. According to social learning theory, Offergelt et al. [37] pointed out that when leaders send a signal of knowledge hiding, subordinates will be encouraged to carry out knowledge hiding behavior. Wei and $\mathrm{Ma}$ [38] used regression analysis to verify the positive influence of leaders' territorial behavior on subordinates' knowledge hiding behavior. Zhai and Shu [39] used an SEM model to verify the negative correlation between a transformational leadership style and knowledge hiding. Men et al. [40] proved the negative correlation between moral leadership and knowledge hiding by regression analysis. He et al. [41] adopted regression analysis to verify that abusive management significantly positively affects knowledge hiding behavior. Yuan et al. [22] proved by regression analysis that there was a significant U-shaped curve relationship between humble leaders and employees' knowledge hiding. In addition, Zhao et al. [42] used regression analysis to verify that the exchange behavior of leaders is negatively correlated with evasive knowledge hiding and deceptive knowledge hiding. Babič et al. [43] proposed that collective prosocial motivation and exchange behavior of leaders and members can reduce knowledge hiding behavior in organizations using the HLM model. Guo et al. [44] established a structural model with Smart PLS 3.0 software and proved the negative influence of knowledge leadership on knowledge hiding intention. In conclusion, positive leadership behavior and style will inhibit the occurrence of knowledge hiding of organizational members, while negative leadership behavior and style will lead to the occurrence of knowledge hiding.

2.4. Individual Traits and Knowledge Hiding. Hernaus et al. [45] demonstrated the positive and significant relationship between individual trait competitiveness and evasive knowledge hiding behavior using regression analysis. Xiong et al. [46] indicated through an inductive analysis that the factors influencing the intention of knowledge hiding come from personal attitudes, perception behavior control, and subjective norms. Butt and Ahmad [47] adopted a multiple case study methodology and pointed out that the reasons for the similar phenomena above are career development, job security, and distrust of competitive organizations from the organizational members themselves. Jha and Varkkey [23] collated and supplemented the interview data with grounded theory and found that other factors affecting knowledge hiding include lack of identification, lack of reciprocity, and lack of confidence. According to Connelly et al. [48], individual trait competitiveness will generate competitive pressure, but surprisingly, the emergence of competitive pressures had no significant impact on the knowledge sharing among organizational members; what really affects knowledge sharing is the stress of busyness caused by low work self-efficacy. In addition, Škerlavaj et al. [49] tested through regression analysis that only when employees' prosocial motivation was low would they feel greater time pressure and thus hide knowledge. In terms of reducing knowledge hiding among organizational members, Geofroy and Evans [50] summarized existing studies and proposed that high-EQ organizational members will enhance cooperation with others and that enhancing mutual trust and organizational commitment can reduce the occurrence of knowledge hiding behavior. Bogilović et al. [51] stated that improved cultural intelligence can enhance the possibility of high-quality social communication among individuals in cultural diversity and buffer the negative relationship between individual knowledge hiding and individual creativity. In conclusion, individual traits are closely related to knowledge hiding, which affects whether an individual is willing and able to share knowledge with others and affects an individual's evaluation of cooperation risks.

\section{Model Description and Assumption}

By performing a systematic literature review of knowledge hiding, Rezwan and Takahashi [52] identified 88 studies that explored different interpersonal, job-related, organizational, individual-related, and knowledge characteristics- 
related variables as reasons for knowledge hiding. This provides an important reference for the construction of the evolutionary game model in this paper. However, this paper intends to establish a universal binary evolutionary game model, so it does not make specific assumptions about the relationship between the game subjects. In addition, considering that job design belongs to the practice of human resource management, job design is summarized to the level of organization and management. Moreover, both explicit knowledge and tacit knowledge have the possibility of knowledge hiding, so this paper does not make specific distinctions on the characteristics of knowledge. In conclusion, this paper holds that the knowledge hiding of organizational members is mainly influenced by organizational management, leadership management, and individual traits. This paper selects variables from the three dimensions to construct an evolutionary game model of knowledge hiding. Lin et al. [53] found that exogenous incentives and distributive justice could reduce knowledge hiding in research project team members' knowledge hiding behaviors. Serenko and Bontis [54] showed that a positive organizational culture reduces the occurrence of knowledge hiding. Malik et al. [55] verified the negative effects of perceptions of organizational politics on employees' creativity. In this paper, motivation factors (positive organizational culture, scientific and reasonable organizational management, etc.) are selected as organizational management variables and incorporated into the evolutionary game model for analysis and are referred to as $e_{i}$.

Jiang et al. [56] considered the possibility that employees are unable to share knowledge and included knowledge ownership in the model for discussion when applying prospect theory to study knowledge hiding. Zhang et al. [57] confirmed in a study on the impact of intellectual property risks on cooperative behavior that once intellectual property risks cause huge losses, enterprises must pay attention to intellectual property risks in the process of cooperation. Mi et al. [58] pointed out that the acquisition, absorption, transformation, and utilization of knowledge all influence the effect of knowledge transfer. The gap in knowledge potential between different subjects plays an important role in the absorption and transformation of knowledge. In this paper, the knowledge stock, knowledge absorption and transformation ability, and knowledge leakage risk of organizational members are selected as individual trait variables to be included in the evolutionary game model for analysis, which are, respectively, represented by $A_{i}, \lambda_{i}$, and $\gamma_{i}$.

Guo et al. [59] verified the negative correlation between knowledge hiding and employee innovation behavior. Yao et al. [60] emphasized in a study on the evolutionary game of knowledge sharing in virtual communities that the collaborative innovation effect can only be generated when two people share knowledge simultaneously. In this paper, collaborative innovation is selected as a leadership management variable to be included in the evolutionary game model for analysis. $\mu_{i}$ represents collaborative innovation ability, and $\beta_{i}$ represents collaborative innovation benefit.
According to the theory of social exchange, what determines a person's social exchange behavior is the reward he gets from others and the cost he pays. Therefore, when pursuing behavioral utility maximization, individuals need to consider the choices of others, which indicates that human beings are bounded by rationality. In this paper, we constructed an evolutionary game model of the knowledge hiding strategies of organizational members based on the assumption of bounded rationality. In accordance with the principle of model simplification, a strategy set is intended to be established in a pure strategy manner. Because evasive knowledge hiding includes knowledge sharing, it is not suitable to be analyzed as pure knowledge hiding. Connelly and Zweig [25] found that rational knowledge hiding does not damage the relationship between organizational members. Therefore, knowledge hiding in this study refers to deceptive knowledge hiding. To make the model easier to understand and clearer, Table 1 summarizes the variables and parameters in this paper.

This section aims to explore the scenario of the dynamic changes of knowledge hiding behavior of organizational members in the process of cooperation. Following the principle of simplicity, we construct a binary knowledge hiding evolutionary game model of organizational members. To better reveal the essence and make the model more reasonable without affecting the conclusion, three assumptions are made as follows.

Assumption 1. Organizational members are bounded by rationality in the process of cooperation and knowledge transaction. Thus, they constantly learn from multiple games and seek the optimal equilibrium strategy with ESS.

Assumption 2. To simplify the evolutionary model, we use $e_{i}$ to represent the various incentives, such as spiritual and material rewards from positive organization management and team leaders and use $C_{i}$ to represent the various costs, such as spiritual and material punishment from negative organization management and team leaders, individual time, and energy. It does not influence the conclusion of this paper.

Assumption 3. Knowledge hiding will harm the organization's interest, but nonknowledge hiding will benefit the organization. Thus, if two organizational members do not intend to hide knowledge, they both gain additional benefits that maximize the interests of both the organization and the members. However, when one party intends to hide knowledge while the other party does not, only the knowledge hiding party gains additional benefits. When both sides choose to hide knowledge, no one gains extra benefits.

Based on the above assumptions and parameter settings, we construct a game model-based payoff function matrix of knowledge hiding, as shown in Table 2, to discuss how the knowledge hiding behavior of organizational members changes dynamically. In this evolutionary model, the respective behavior strategy sets of organizational members are $\{$ knowledge hiding, nonknowledge hiding\}, in which we 
TABle 1: Description of parameters.

\begin{tabular}{lc}
\hline Parameters & Description \\
\hline$S_{i}$ & The direct benefit when organizational members choose knowledge hiding $S_{i},(i=1,2)$ \\
$A_{i}$ & The knowledge stock of one organizational member $A_{i},(i=1,2)$ \\
$\lambda_{i}$ & The coefficient of knowledge absorption and transformation ability $\lambda_{i},(i=1,2)(0<\lambda i<1)$ \\
$\alpha_{i}$ & The coefficient of direct benefit $\alpha_{i},(i=1,2)(0<\alpha i<1)$ \\
$\mu_{i}$ & The coefficient of collaborative innovation ability $\mu_{i},(i=1,2)(0<\mu i<1)$ \\
$\beta_{i}$ & The coefficient of collaborative innovation benefit $\beta_{i},(i=1,2)(0<\beta i<1)$ \\
$\gamma_{i}$ & The coefficient of knowledge leakage risk $\gamma_{i},(0<\gamma i<1)$ \\
$C_{i}$ & The cost of nonknowledge hiding behavior $C_{i},(i=1,2)\left(C_{i}>0\right)$ \\
$e_{i}$ & Incentives coming from the organization and team leaders $e_{i},\left(0<e_{i}<1\right)$ \\
$x$ & The probability of knowledge hiding of member $1 x(0 \leq x \leq 1)$ \\
$y$ & The probability of knowledge hiding of member $2 y(0 \leq y \leq 1)$ \\
\hline
\end{tabular}

TABLE 2: Game model-based payoff function matrix of knowledge hiding.

\begin{tabular}{lcc}
\hline & $\begin{array}{c}\text { Member } 2 \\
\text { Knowledge hiding } \\
(y)\end{array}$ & $\begin{array}{c}\text { Member 2 } \\
\text { Nonknowledge hiding } \\
(1-y)\end{array}$ \\
\hline Member 1 & $S_{1}$ & $S_{1}+\lambda_{1} A_{2} \alpha_{1}$ \\
Knowledge hiding $(x)$ & $S_{2}$ & $-\gamma_{2} \lambda_{1} A_{2} \alpha_{1}-C_{2}+e_{2} A_{2}$ \\
Member 1 & $-\gamma_{1} \lambda_{2} A_{1} \alpha_{2}-C_{1}+e_{1} A_{1}$ & $\lambda_{1} A_{2} \alpha_{1}+\mu_{1}\left(A_{1}+A_{2}\right) \beta_{1}-\gamma_{1} \lambda_{2} A_{1} \alpha_{2}-C_{1}+e_{1} A_{1}$ \\
Nonknowledge hiding $(1-x)$ & $S_{2}+\lambda_{2} A_{1} \alpha_{2}$ & $\lambda_{2} A_{1} \alpha_{2}+\mu_{2}\left(A_{1}+A_{2}\right) \beta_{2}-\gamma_{2} \lambda_{1} A_{2} \alpha_{1}-C_{2}+e_{2} A_{2}$ \\
\hline
\end{tabular}

use $\{x,(1-x)\}$ to represent the knowledge hiding probability sets of member 1 and $\{y,(1-y)\}$ to represent the knowledge hiding probability sets of member 2 .

\section{Evolutionary Game Analysis Based on the Replication Dynamic Equation}

4.1. Evolutionary Game Analysis of Member 1. According to the replicator dynamic equation, the expected benefits of knowledge hiding in the two members of the organization are obtained. Whether member 1 is in the state of selecting collaboration or not, the expected benefits obtained by individuals are as follows.

When member 1 chooses to hide his knowledge, the expected benefit is

$$
U_{11}=y S_{1}+(1-y)\left(S_{1}+\lambda_{1} A_{2} \alpha_{1}\right)
$$

When member 1 chooses not to hide knowledge, the expected benefit is

$$
U_{12}=y\left[-\gamma_{1} \lambda_{2} A_{1} \alpha_{2}-C_{1}+e_{1} A_{1}\right]+(1-y)\left[\lambda_{1} A_{2} \alpha_{1}+\mu_{1}\left(A_{1}+A_{2}\right) \beta_{1}-\gamma_{1} \lambda_{2} A_{1} \alpha_{2}-C_{1}+e_{1} A_{1}\right] .
$$

Then, the average expected benefit of member 1 is as follows:

$$
\bar{U}_{1}=x U_{11}+(1-x) U_{12} .
$$

Thus, the replication dynamic equation of member 1 adopting the knowledge hiding strategy changing with time can be expressed as follows:

$$
\begin{aligned}
F(x) & =\frac{\mathrm{d} x}{\mathrm{~d} t} \\
& =x\left(U_{11}-\bar{U}_{1}\right) \\
& =x(1-x)\left(U_{11}-U_{12}\right) \\
& =x(1-x)\left[(y-1) \mu_{1}\left(A_{1}+A_{2}\right) \beta_{1}+S_{1}+\gamma_{1} \lambda_{2} A_{1} \alpha_{2}+C_{1}-e_{1} A_{1}\right] .
\end{aligned}
$$


The process of strategy evolution adjustment analysis is based on the comparison between the value of the game benefit function of knowledge hiding and nonknowledge hiding under a stable evolution state. According to equation (4), $F^{\prime}(x)$ is shown in the following equation:

$$
F^{\prime}(x)=(1-2 x)\left[(y-1) \mu_{1}\left(A_{1}+A_{2}\right) \beta_{1}+S_{1}+\gamma_{1} \lambda_{2} A_{1} \alpha_{2}+C_{1}-e_{1} A_{1}\right] .
$$

To obtain the stable strategy of the evolutionary game, we need to find the equilibrium point of the replication dynamic equation, and the equilibrium point $x^{*}$ needs to meet $F\left(x^{*}\right)=0$ and $F^{\prime}\left(x^{*}\right)<0$. Then, if $F(x)=0$, the solution of equation (4) is $x^{*}=0, x^{*}=1, y^{*}=\left(\left(\mu_{1}\left(A_{1}+\right.\right.\right.$ $\left.\left.\left.A_{2}\right) \beta_{1}-S_{1}-\gamma_{1} \lambda_{2} A_{1} \alpha_{2}-C_{1}+e_{1} A_{1}\right) /\left(\mu_{1}\left(A_{1}+A_{2}\right) \beta_{1}\right)\right)$. Thus, we know how the stable strategy of member 1 for knowledge hiding or nonknowledge hiding dynamic changes with the changing of relative variables.

(i) When $y^{*}=\left(\left(\mu_{1}\left(A_{1}+A_{2}\right) \beta_{1}-S_{1}-\gamma_{1} \lambda_{2} A_{1} \alpha_{2}-C_{1}\right.\right.$ $\left.\left.+e_{1} A_{1}\right) /\left(\mu_{1}\left(A_{1}+A_{2}\right) \beta_{1}\right)\right), \quad F(x)=0$ under the condition of $\forall x$. At this time, it is a stable equilibrium strategy.

(ii) When $y^{*} \neq\left(\left(\mu_{1}\left(A_{1}+A_{2}\right) \beta_{1}-S_{1}-\gamma_{1} \lambda_{2} A_{1} \alpha_{2}-C_{1}+\right.\right.$ $\left.\left.e_{1} A_{1}\right) / \mu_{1}\left(A_{1}+A_{2}\right) \beta_{1}\right)$, the stable equilibrium strategy could be $x^{*}=0$ or $x^{*}=1$. According to equation (5), if $S_{1}+\gamma_{1} \lambda_{2} A_{1} \alpha_{2}+C_{1}-e_{1} A_{1}>\mu_{1}\left(A_{1}+A_{2}\right) \beta_{1}$,
$F^{\prime}\left(x^{*}=1\right)<0$ and $F^{\prime}\left(x^{*}=0\right)>0$. Thus, the stable point is $x^{*}=1$, so member 1 is willing to hide the knowledge. However, if $S_{1}+\gamma_{1} \lambda_{2} A_{1} \alpha_{2}+C_{1}-e_{1} A_{1}$ $<\mu_{1}\left(A_{1}+A_{2}\right) \beta_{1}$ and $F^{\prime}\left(x^{*}=1\right)<0$ when $y>y^{*}$, the stable strategy of member 1 is knowledge hiding, as the stable point is $x^{*}=1$, and when $y<y^{*}$, $F^{\prime}\left(x^{*}=0\right)<0$, the stable strategy of member 1 is nonknowledge hiding, as the stable point is $x^{*}=0$.

The above situations illustrate that the dynamic evolution of the stable strategy for member 1 is influenced by both the probability of knowledge hiding of member $2(y)$ and the critical value $\left(y^{*}\right)$. When $y>y^{*}$, the evolutionarily stable strategy of member 1 hides knowledge. However, when $y<y^{*}$, the evolutionarily stable strategy of member 1 is nonknowledge hiding.

4.2. Evolutionary Game Analysis of Member 2. Similarly, when member 2 is in the state of selecting collaboration or not, the expected benefits obtained are as follows.

When member 2 chooses to hide his knowledge, the expected benefit is

$$
U_{21}=x S_{2}+(1-x)\left(S_{2}+\lambda_{2} A_{1} \alpha_{2}\right)
$$

When member 2 chooses not to hide knowledge, the expected benefit is

$$
U_{22}=x\left[-\gamma_{2} \lambda_{1} A_{2} \alpha_{1}-C_{2}+e_{2} A_{2}\right]+(1-x)\left[\lambda_{2} A_{1} \alpha_{2}+\mu_{2}\left(A_{1}+A_{2}\right) \beta_{2}-\gamma_{2} \lambda_{1} A_{2} \alpha_{1}-C_{2}+e_{2} A_{2}\right] .
$$
follows:

Then, the average expected benefit of member 2 is as

$$
\bar{U}_{2}=y U_{21}+(1-y) U_{22} .
$$

Thus, the replication dynamic equation of member 2 choosing the knowledge hiding strategy changing with time can be expressed as follows:

$$
\begin{aligned}
F(y) & =\frac{d y}{d t} \\
& =y\left(U_{21}-\bar{U}_{2}\right) \\
& =y(1-y)\left(U_{21}-U_{22}\right) \\
& =y(1-y)\left[(x-1) \mu_{2}\left(A_{1}+A_{2}\right) \beta_{2}+S_{2}+\gamma_{2} \lambda_{1} A_{2} \alpha_{1}+C_{2}-e_{2} A_{2}\right] .
\end{aligned}
$$

According to equation (9), $F^{\prime}(y)$ is shown in the following equation:

$$
F^{\prime}(y)=(1-2 y)\left[(x-1) \mu_{2}\left(A_{1}+A_{2}\right) \beta_{2}+S_{2}+\gamma_{2} \lambda_{1} A_{2} \alpha_{1}+C_{2}-e_{2} A_{2}\right]
$$

To obtain the stable strategy of the evolutionary game, we need to find the equilibrium point of the replication dynamic equation, and the equilibrium point $y^{*}$ needs to meet $F\left(y^{*}\right)=0$ and $F^{\prime}\left(y^{*}\right)<0$. Then, let $F(y)=0$, and the solution of equation (9) is $y^{*}=0, y^{*}=1, x^{*}=\left(\left(\mu_{2}\left(A_{1}+\right.\right.\right.$ $\left.\left.\left.A_{2}\right) \beta_{2}-S_{2}-\gamma_{2} \lambda_{1} A_{2} \alpha_{1}-C_{2}+e_{2} A_{2}\right) /\left(\mu_{2}\left(A_{1}+A_{2}\right) \beta_{2}\right)\right)$. We 
know how the stable strategy of member 2 for knowledge hiding or nonknowledge hiding dynamic changes with the changing of relative variables.

(i) When $x^{*}=\left(\left(\mu_{2}\left(A_{1}+A_{2}\right) \beta_{2}-S_{2}-\gamma_{2} \lambda_{1} A_{2} \alpha_{1}-C_{2}\right.\right.$ $\left.\left.+e_{2} A_{2}\right) /\left(\mu_{2}\left(A_{1}+A_{2}\right) \beta_{2}\right)\right), \quad F(y)=0$ under the condition of $\forall y$. At this time, it is a stable equilibrium strategy.

(ii) When $x^{*} \neq\left(\left(\mu_{2}\left(A_{1}+A_{2}\right) \beta_{2}-S_{2}-\gamma_{2} \lambda_{1} A_{2} \alpha_{1}-C_{2}\right.\right.$ $\left.\left.+e_{2} A_{2}\right) /\left(\mu_{2}\left(A_{1}+A_{2}\right) \beta_{2}\right)\right)$, the stable equilibrium strategy could be $y^{*}=0$ or $y^{*}=1$. According to equation (10), if $S_{2}+\gamma_{2} \lambda_{1} A_{2} \alpha_{1}+C_{2}-e_{2} A_{2}>\mu_{2}\left(A_{1}\right.$ $\left.+A_{2}\right) \beta_{2}, F^{\prime}\left(y^{*}=1\right)<0$, and $F^{\prime}\left(y^{*}=0\right)>0$. Thus, the stable point is $y^{*}=1$, so member 2 is willing to hide the knowledge. However, if $S_{2}+\gamma_{2} \lambda_{1} A_{2} \alpha_{1}+$ $C_{2}-e_{2} A_{2}<\mu_{2}\left(A_{1}+A_{2}\right) \beta_{2}, \quad F^{\prime}\left(y^{*}=1\right)<0 \quad$ when $x>x^{*}$, the stable strategy of member 2 is knowledge hiding, as the stable point is $y^{*}=1$, and when $x<x^{*}$, $F^{\prime}\left(y^{*}=0\right)<0$, the stable strategy of member 2 is nonknowledge hiding, as the stable point is $y^{*}=0$.

The above situations illustrate that the ESS for member 2 is influenced by both the probability of knowledge hiding of member $1(x)$ and the critical value of $\left(x^{*}\right)$. When $x>x^{*}$, the evolutionarily stable strategy of member 2 is to hide knowledge, but when $x<x^{*}$, the evolutionarily stable strategy of member 2 is not to hide knowledge.

4.3. Evolutionary Game Analysis of Both Members 1 and 2. The above sections discuss the ESS of member 1 and member 2 in the organization. However, because the knowledge hiding behaviors of organizational members are affected by the other members, the situation of the organization is constantly changing. Thus, this subsection uses the replicator dynamics equations of both groups to obtain the equilibrium solution and analyzes the stable solution of the model using the equilibrium solution and the Jacobian matrix.

Let $M_{1}=\mu_{1}\left(A_{1}+A_{2}\right) \beta_{1}, N_{1}=\mu_{1}\left(A_{1}+A_{2}\right) \beta_{1}-S_{1}-\gamma_{1}$ $\lambda_{2} A_{1} \alpha_{2}-C_{1}+e_{1} A_{1}$, and $M_{2}=\mu_{2}\left(A_{1}+A_{2}\right) \beta_{2}, N_{2}=\mu_{2}\left(A_{1}\right.$ $\left.+A_{2}\right) \beta_{2}-S_{2}-\gamma_{2} \lambda_{1} A_{2} \alpha_{1}-C_{2}+e_{2} A_{2}$. The Jacobian matrix of this evolutionary game model is shown in equation (11), and the determinant and trace of (11) can be calculated as (12) and (13), respectively:

$$
\begin{aligned}
& J=\left(\begin{array}{ll}
\frac{\partial F(x)}{\partial x} & \frac{\partial F(x)}{\partial y} \\
\frac{\partial F(y)}{\partial x} & \frac{\partial F(y)}{\partial y}
\end{array}\right) \\
& =\left(\begin{array}{cc}
(1-2 x)\left(y M_{1}-N_{1}\right) & x(1-x) M_{1} \\
y(1-y) M_{2} & (1-2 y)\left(x M_{2}-N_{2}\right)
\end{array}\right), \\
& \operatorname{De} t(J)=\frac{\partial F(x)}{\partial x} \frac{\partial F(y)}{\partial y}-\frac{\partial F(x)}{\partial y} \frac{\partial F(y)}{\partial x} \\
& =(1-2 x)\left(y M_{1}-N_{1}\right)(1-2 y)\left(x M_{2}-N_{2}\right)-x(1-x) M_{1} y(1-y) M_{2}, \\
& \operatorname{Tr}(J)=\frac{\partial F(x)}{\partial x}-\frac{\partial F(y)}{\partial y} \\
& =(1-2 x)\left(y M_{1}-N_{1}\right)+(1-2 y)\left(x M_{2}-N_{2}\right) .
\end{aligned}
$$

Scenario 1. When $M_{1}<N_{1}$ and $M_{2}<N_{2}$, there are five equilibrium solutions in the equation group of knowledge hiding among two organizational members, which is composed of $F(x)$ and $F(y): A(0,0), B(1,0), C(1,1)$, $D(0,1), E\left(x^{*}, y^{*}\right)$. The stability analysis results of these five equilibrium points are shown in Table 3.

According to Table 3, the stable strategy of the evolutionary game system can be drawn, as shown in Figure 1. As the probability of knowledge hiding decreases, the evolutionary game system converges to point $A(0,0)$ so that the stable strategy of the evolutionary game system of two members is nonknowledge hiding.
Scenario 2. When $M_{1}<N_{1}$ and $M_{2}>N_{2}$, there are also five equilibrium solutions in the equation group of knowledge hiding, constituted by $F(x)$ and $F(y): A(0,0), B(1,0)$, $C(1,1), D(0,1), E\left(x^{*}, y^{*}\right)$. The stability analysis results of these five equilibrium points are shown in Table 4 .

According to Table 4, we can draw the stable strategy of the evolutionary game system of the two members in Figure 2. The benefits of member 1 choosing the nonknowledge hiding strategy are superior to those of member 1 choosing the knowledge hiding strategy. However, for member 2, the choice of strategy for knowledge hiding or nonknowledge hiding depends on the size of $x^{*}$. When $x>x^{*}$, member 2 
TABLE 3: Stability analysis of equilibrium points when $M_{1}<N_{1}$ and $M_{2}<N_{2}$.

\begin{tabular}{|c|c|c|c|c|c|}
\hline $\begin{array}{l}\text { Equilibrium } \\
\text { solution }\end{array}$ & $\operatorname{Det}(J)$ & $\begin{array}{c}\text { Sign } \\
\text { symbol }\end{array}$ & $\operatorname{Tr}(J)$ & $\begin{array}{c}\text { Sign } \\
\text { symbol }\end{array}$ & $\begin{array}{l}\text { Judgement } \\
\text { result }\end{array}$ \\
\hline $\begin{array}{l}A(0,0) \\
B(1,0) \\
C(1,1) \\
D(0,1) \\
E\left(x^{*}, y^{*}\right) \\
\end{array}$ & $\begin{array}{c}N_{1} N_{2} \\
N_{1}\left(M_{2}-N_{2}\right) \\
\left(N_{1}-M_{1}\right)\left(N_{2}-M_{2}\right) \\
\left(M_{1}-N_{1}\right) N_{2} \\
-N_{1} N_{2} M_{1} M_{2}\left(M_{1}-N_{1}\right)\left(M_{2}-N_{2}\right) / M_{1}^{2} M_{2}^{2}\end{array}$ & $\begin{array}{l}- \\
- \\
+ \\
- \\
-\end{array}$ & $\begin{array}{c}-\left(N_{1}+N_{2}\right) \\
N_{1}+\left(M_{2}-N_{2}\right) \\
\left(N_{1}-M_{1}\right)+\left(N_{2}-M_{2}\right) \\
\left(M_{1}-N_{1}\right)+N_{2} \\
0\end{array}$ & $\begin{array}{c}- \\
\text { Uncertain } \\
+ \\
\text { Uncertain } \\
\text { Uncertain }\end{array}$ & $\begin{array}{c}\text { ESS } \\
\text { Saddle point } \\
\text { Unstable point } \\
\text { Saddle point } \\
\text { Saddle point }\end{array}$ \\
\hline
\end{tabular}

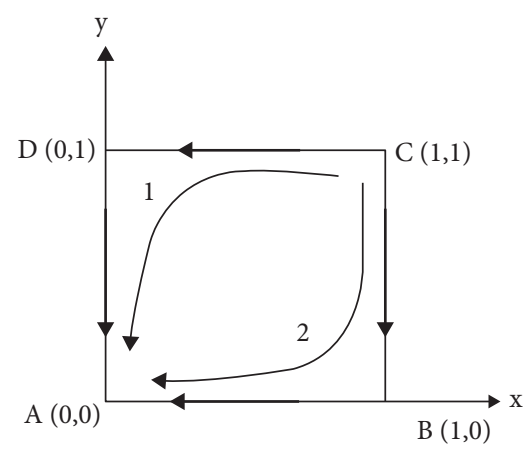

Figure 1: The stable strategy of the evolutionary game system when $M_{1}<N_{1}$ and $M_{2}<N_{2}$.

TABLE 4: Stability analysis of equilibrium points when $M_{1}<N_{1}$ and $M_{2}>N_{2}$.

\begin{tabular}{|c|c|c|c|c|c|}
\hline $\begin{array}{l}\text { Equilibrium } \\
\text { solution }\end{array}$ & $\operatorname{De} t(J)$ & $\begin{array}{c}\text { Sign } \\
\text { symbol }\end{array}$ & $\operatorname{Tr}(J)$ & $\begin{array}{c}\text { Sign } \\
\text { symbol }\end{array}$ & $\begin{array}{l}\text { Judgement } \\
\text { result }\end{array}$ \\
\hline $\begin{array}{l}A(0,0) \\
B(1,0) \\
C(1,1) \\
D(0,1) \\
E\left(x^{*}, y^{*}\right)\end{array}$ & $\begin{array}{c}N_{1} N_{2} \\
N_{1}\left(M_{2}-N_{2}\right) \\
\left(N_{1}-M_{1}\right)\left(N_{2}-M_{2}\right) \\
\left(M_{1}-N_{1}\right) N_{2} \\
-N_{1} N_{2} M_{1} M_{2}\left(M_{1}-N_{1}\right)\left(M_{2}-N_{2}\right) / M_{1}^{2} M_{2}^{2}\end{array}$ & $\begin{array}{l}+ \\
+ \\
- \\
- \\
+\end{array}$ & $\begin{array}{c}-\left(N_{1}+N_{2}\right) \\
N_{1}+\left(M_{2}-N_{2}\right) \\
\left(N_{1}-M_{1}\right)+\left(N_{2}-M_{2}\right) \\
\left(M_{1}-N_{1}\right)+N_{2} \\
0\end{array}$ & $\begin{array}{c}- \\
+ \\
\text { Uncertain } \\
\text { Uncertain } \\
\text { Uncertain }\end{array}$ & $\begin{array}{l}\text { ESS } \\
\text { Unstable point } \\
\text { Saddle point } \\
\text { Saddle point } \\
\text { Saddle point }\end{array}$ \\
\hline
\end{tabular}

tends to choose the knowledge hiding strategy. When $x<x^{*}$, the probability of knowledge hiding of member 2 gradually decreases, and they gradually tend to choose nonknowledge hiding. In this scenario, the evolutionary game system of both sides converges to the point $A(0,0)$, and the stable strategy of the evolutionary game system for two members is (nonknowledge hiding, nonknowledge hiding).

Scenario 3. When $M_{1}>N_{1}$ and $M_{2}<N_{2}$, there are also five equilibrium solutions in the equation group of knowledge hiding, which is constituted by $F(x)$ and $F(y): A(0,0)$, $B(1,0), C(1,1), D(0,1), E\left(x^{*}, y^{*}\right)$. The stability analysis results of these five equilibrium points are shown in Table 5.

According to Table 5, the benefit of member 2 choosing the nonknowledge hiding strategy is superior to that of the knowledge hiding strategy. However, for member 1, the choice of strategy for knowledge hiding or nonknowledge hiding depends on the size of $y^{*}$. When $y>y^{*}$, member 1 tends to choose the knowledge hiding strategy; when $y<y^{*}$, the probability of knowledge hiding of member 1 decreases gradually, and he gradually tends to choose a nonknowledge hiding strategy. At this time, the evolutionary game system of both sides converges to point $A(0,0)$, and the stable strategy of the evolutionary game system is (nonknowledge hiding, nonknowledge hiding), as shown in Figure 3.

Scenario 4. When $M_{1}>N_{1}$ and $M_{2}>N_{2}$, there are five equilibrium solutions in the group of knowledge hiding, which is constituted by $F(x)$ and $F(y): A(0,0), B(1,0)$, $C(1,1), D(0,1), E\left(x^{*}, y^{*}\right)$. The stability analysis results of these five equilibrium points are shown in Table 6 .

As shown in Table 6 , there are two stable equilibrium strategies for member 1 and member 2: $A(0,0)$ and $E\left(x^{*}, y^{*}\right)$. At this point, both sides' choice of strategy depends not only on the initial probability of strategy selection but also on $\left(x^{*}, y^{*}\right)$, that is,

$$
\left(x^{*}=\frac{\mu_{2}\left(A_{1}+A_{2}\right) \beta_{2}-S_{2}-\gamma_{2} \lambda_{1} A_{2} \alpha_{1}-C_{2}+e_{2} A_{2}}{\mu_{2}\left(A_{1}+A_{2}\right) \beta_{2}}, y^{*}=\frac{\mu_{1}\left(A_{1}+A_{2}\right) \beta_{1}-S_{1}-\gamma_{1} \lambda_{2} A_{1} \alpha_{2}-C_{1}+e_{1} A_{1}}{\mu_{1}\left(A_{1}+A_{2}\right) \beta_{1}}\right) .
$$




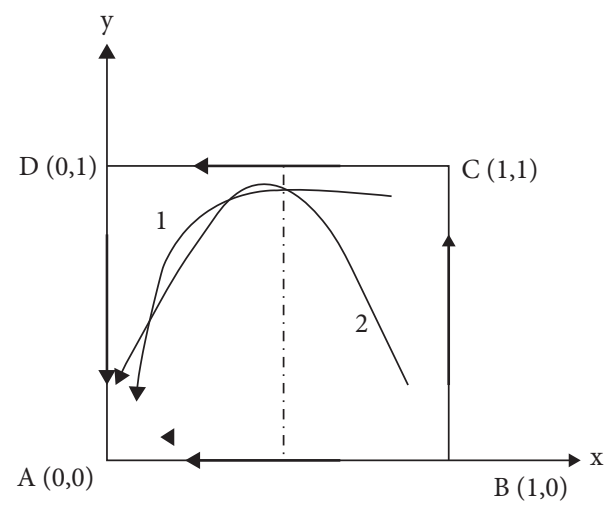

FIgURE 2: The stable strategy of the evolutionary game system when $M_{1}<N_{1}$ and $M_{2}>N_{2}$.

TABLE 5: Stability analysis of equilibrium points when $M_{1}>N_{1}$ and $M_{2}<N_{2}$.

\begin{tabular}{|c|c|c|c|c|c|}
\hline $\begin{array}{l}\text { Equilibrium } \\
\text { solution }\end{array}$ & $\operatorname{Det}(J)$ & $\begin{array}{l}\text { Sign } \\
\text { symbol }\end{array}$ & $\operatorname{Tr}(J)$ & $\begin{array}{c}\text { Sign } \\
\text { symbol }\end{array}$ & $\begin{array}{l}\text { Judgement } \\
\text { result }\end{array}$ \\
\hline$A(0,0)$ & $N_{1} N_{2}$ & + & $-\left(N_{1}+N_{2}\right)$ & - & ESS \\
\hline$B(1,0)$ & $N_{1}\left(M_{2}-N_{2}\right)$ & - & $N_{1}+\left(M_{2}-N_{2}\right)$ & Uncertain & Saddle point \\
\hline$C(1,1)$ & $\left(N_{1}-M_{1}\right)\left(N_{2}-M_{2}\right)$ & - & $\left(N_{1}-M_{1}\right)+\left(N_{2}-M_{2}\right)$ & Uncertain & Saddle point \\
\hline$D(0,1)$ & $\left(M_{1}-N_{1}\right) N_{2}$ & + & $\left(M_{1}-N_{1}\right)+N_{2}$ & + & Unstable point \\
\hline$E\left(x^{*}, y^{*}\right)$ & $-N_{1} N_{2} M_{1} M_{2}\left(M_{1}-N_{1}\right)\left(M_{2}-N_{2}\right) / M_{1}^{2} M_{2}^{2}$ & + & 0 & Uncertain & Saddle point \\
\hline
\end{tabular}

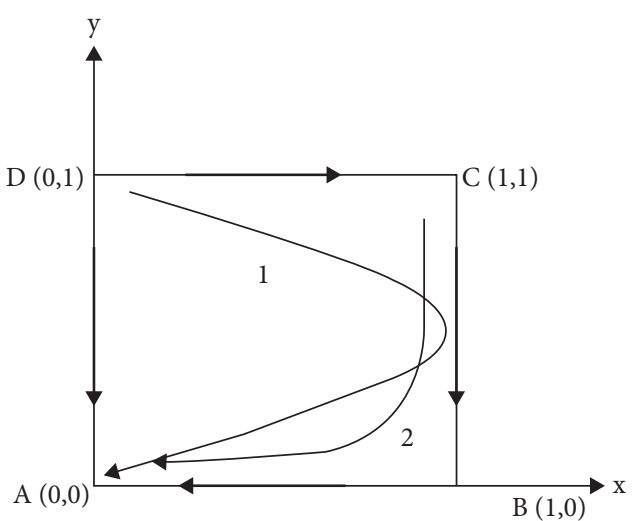

FIgURE 3: The stable strategy of the evolutionary game system when $M_{1}>N_{1}$ and $M_{2}<N_{2}$.

As team members continue to learn and improve, the evolutionary game process is shown in Figure 4. In region $\mathrm{DABE}$, the knowledge hiding probabilities of member 1 and member 2 decrease, and then the evolutionary game system of both sides converges to point $A(0,0)$, at which the stable equilibrium strategy of the evolutionary game is (nonknowledge hiding, nonknowledge hiding). In region $\mathrm{DEBC}$, the nonknowledge hiding probabilities of member 1 and member 2 decrease, and then the evolutionary game system of both sides converges to point $C(1,1)$, at which the stable equilibrium strategy of the evolutionary game is (knowledge hiding, knowledge hiding). We use $S_{\mathrm{DABE}}=(1 / 2)\left(x^{*}+\right.$ $\left.y^{*}\right)$ to represent the area of DABE. When $x^{*}$ and $y^{*}$ decrease, $S_{\mathrm{DABE}}$ decreases and $S_{\mathrm{DEBC}}$ increases, so the probability of knowledge hiding increases, and then the stable point is $C(1,1)$. In contrast, when $x^{*}$ and $y^{*}$ become larger, $S_{\mathrm{DABE}}$ becomes larger but $S D E B C$ decreases so that the possibility of nonknowledge hiding increases gradually and then the stable point approaches $A(0,0)$.

\section{Numerical Simulation and Results}

In this section, a numerical simulation is conducted to analyze the knowledge hiding strategies among organizational members under the effects of relevant factors, which can provide a clear and straightforward perspective. The above analysis results show that the saddle point and the stable equilibrium point are influenced by both initial values and the changes in relevant parameters. Here, we set initial parameters to conduct sensitivity analysis to changes in parameters on the equilibrium strategy of knowledge hiding in Table 7.

5.1. Effect of Knowledge Leakage Risk. We further analyze how the knowledge leakage risk affects the knowledge hiding behavior of organizational members in Figure 5. Knowledge leakage risk is positively correlated with knowledge hiding behavior. With the risk of knowledge leakage steadily increasing, the value for the saddle point decreases, resulting in a higher probability that the stable equilibrium point converges to $C(1,1)$. Thus, the possibility for organizational members choosing knowledge hiding behavior becomes greater. Organizational members share their knowledge without reservations, which may threaten personal career development and job security. It may weaken the knowledge power of the members of the organization and threaten their status in the organization. If the organization lacks fair management and other partners do not have the knowledge, the leader may 
TABLE 6: Stability analysis of equilibrium points when $M_{1}>N_{1}$ and $M_{2}>N_{2}$.

\begin{tabular}{|c|c|c|c|c|c|}
\hline $\begin{array}{l}\text { Equilibrium } \\
\text { solution }\end{array}$ & $\operatorname{Det}(J)$ & $\begin{array}{c}\text { Sign } \\
\text { symbol }\end{array}$ & $\operatorname{Tr}(J)$ & $\begin{array}{c}\text { Sign } \\
\text { symbol }\end{array}$ & $\begin{array}{l}\text { Judgement } \\
\text { result }\end{array}$ \\
\hline$A(0,0)$ & $N_{1} N_{2}$ & + & $-\left(N_{1}+N_{2}\right)$ & - & ESS \\
\hline$B(1,0)$ & $N_{1}\left(M_{2}-N_{2}\right)$ & + & $N_{1}+\left(M_{2}-N_{2}\right)$ & + & Unstable point \\
\hline$C(1,1)$ & $\left(N_{1}-M_{1}\right)\left(N_{2}-M_{2}\right)$ & + & $\left(N_{1}-M_{1}\right)+\left(N_{2}-M_{2}\right)$ & - & ESS \\
\hline$D(0,1)$ & $\left(M_{1}-N_{1}\right) N_{2}$ & + & $\left(M_{1}-N_{1}\right)+N_{2}$ & + & Unstable point \\
\hline$E\left(x^{*}, y^{*}\right)$ & $-N_{1} N_{2} M_{1} M_{2}\left(M_{1}-N_{1}\right)\left(M_{2}-N_{2}\right) / M_{1}^{2} M_{2}^{2}$ & - & 0 & Uncertain & Saddle point \\
\hline
\end{tabular}

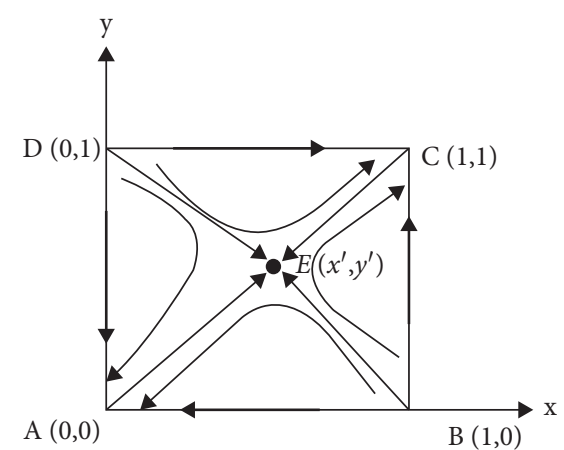

Figure 4: The stable strategy of the evolutionary game system when $M_{1}>N_{1}$ and $M_{2}>N_{2}$.

TABLE 7: Initial values of relevant parameters.

\begin{tabular}{lcccccccccc}
\hline Parameters & $S_{1}$ & $A_{1}$ & $\lambda_{1}$ & $\alpha_{1}$ & $\mu_{1}$ & $\beta_{1}$ & $\gamma_{1}$ & $C_{1}$ & $e_{1}$ & $x$ \\
\hline Value & 100 & 100 & 0.8 & 0.4 & 0.6 & 0.3 & 0.5 & 70 & 0.4 & 0.6 \\
\hline Parameters & $S_{2}$ & $A_{2}$ & $\lambda_{2}$ & $\alpha_{2}$ & $\mu_{2}$ & $\beta_{2}$ & $\gamma_{2}$ & $C_{2}$ & $e_{2}$ & $y$ \\
\hline Value & 80 & 80 & 0.6 & 0.2 & 0.4 & 0.1 & 0.3 & 50 & 0.3 & 0.55 \\
\hline
\end{tabular}

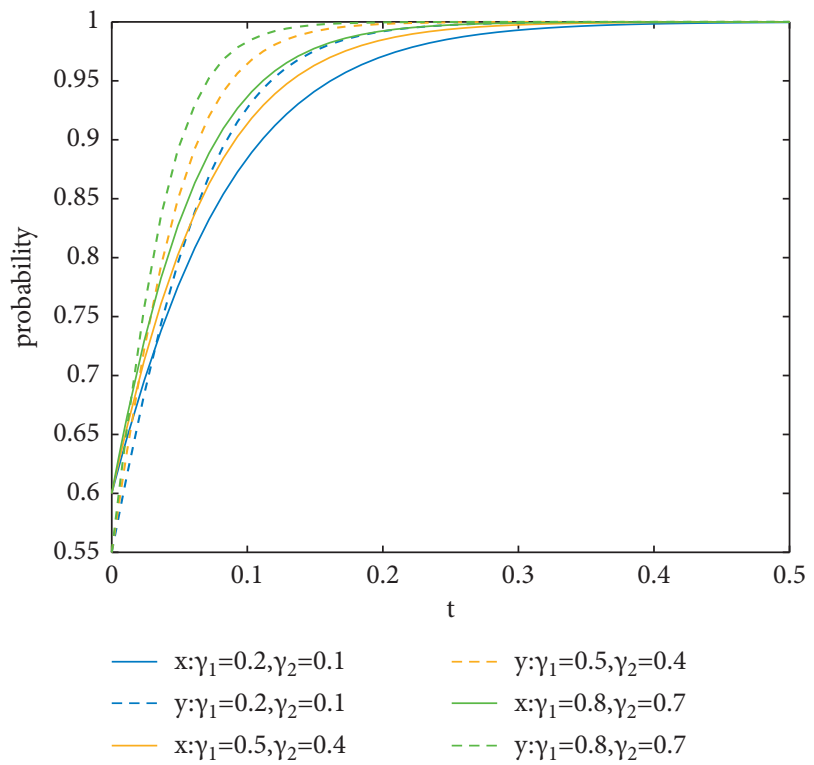

FIGURE 5: The effect of knowledge leakage risk on knowledge hiding.

assign more work to himself. Therefore, when organizational members realize that the knowledge leakage risk gradually increases, they increase the possibility of knowledge hiding.
5.2. Effect of Knowledge Absorption and Transformation Ability. The knowledge absorption and transformation ability of organizational members is an additional factor affecting knowledge hiding behavior. Figure 6 shows how the knowledge hiding behavior of organizational members changes when enhancing the knowledge absorption and transformation capacity and keeping other parameters constant. Knowledge absorption and transformation ability is positively correlated with knowledge hiding behavior. As the knowledge absorption and transformation capacity gradually increases, the value for the saddle point decreases, resulting in a higher probability that the stable equilibrium point converges to $C(1,1)$. Thus, the possibility of both members choosing a knowledge hiding strategy is increased. The high-intelligence group in the organization has stronger knowledge transformation and absorption ability compared with others. Just absorbing and transforming other people's knowledge and hiding one's own knowledge can maximize personal benefits, but obviously, such behavior is not conducive to the cooperation and innovation development of the organization.

5.3. Effect of Collaborative Innovation Ability. As shown in Figure 7, collaborative innovation ability is correlated with knowledge hiding behavior. When the collaborative innovation ability is poor, the value for the saddle point decreases with increasing $\mu 1$, resulting in a larger probability that the stable equilibrium point converges to $C(1,1)$. Thus, the possibility of both members choosing a knowledge hiding strategy increases. However, when collaborative innovation ability is stronger, the possibility of both members choosing a knowledge hiding strategy increases less significantly with increasing $\mu_{1}$. When the collaborative innovation ability of the organizational members is weak, the innovation results cannot be produced even if the knowledge is shared. In this case, the members would rather choose knowledge hiding to save opportunity costs. However, when organizational members have strong innovation ability, there is a possibility to produce innovative results through cooperation. Then, the curve of the occurrence probability of the members' knowledge hiding behavior is not as steep as that in the previous situation.

5.4. Effect of Knowledge Stock. To discuss how the stock of knowledge affects knowledge hiding behavior, we provide intuitive insight into the specific trend in Figure 8. When the stock of knowledge is low, the saddle point decreases, and 


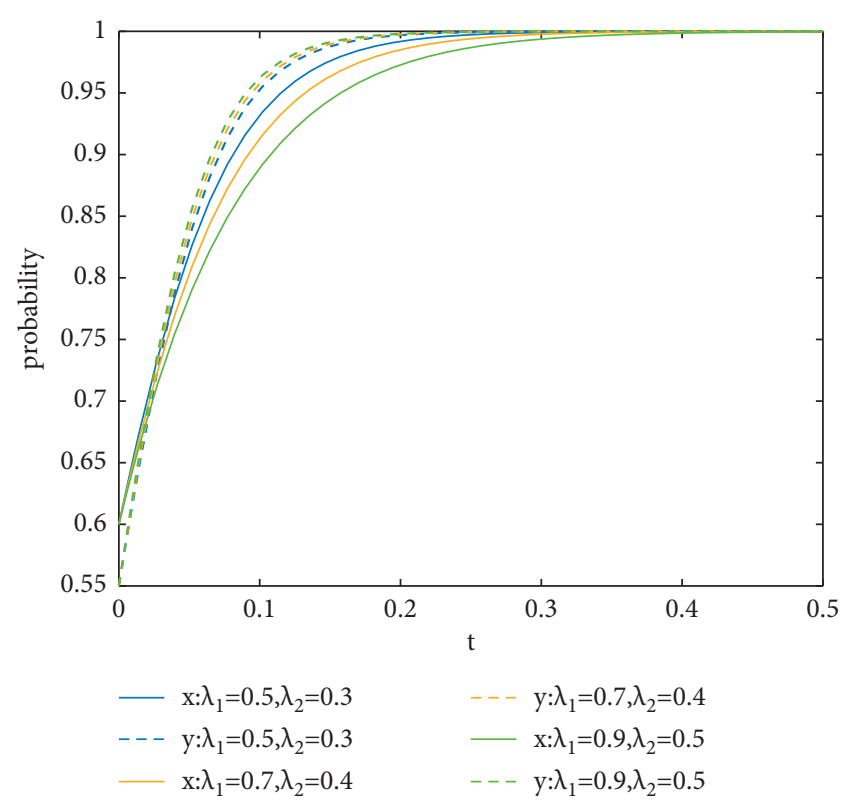

FIGURE 6: The effects of knowledge absorption and transformation ability on knowledge hiding.

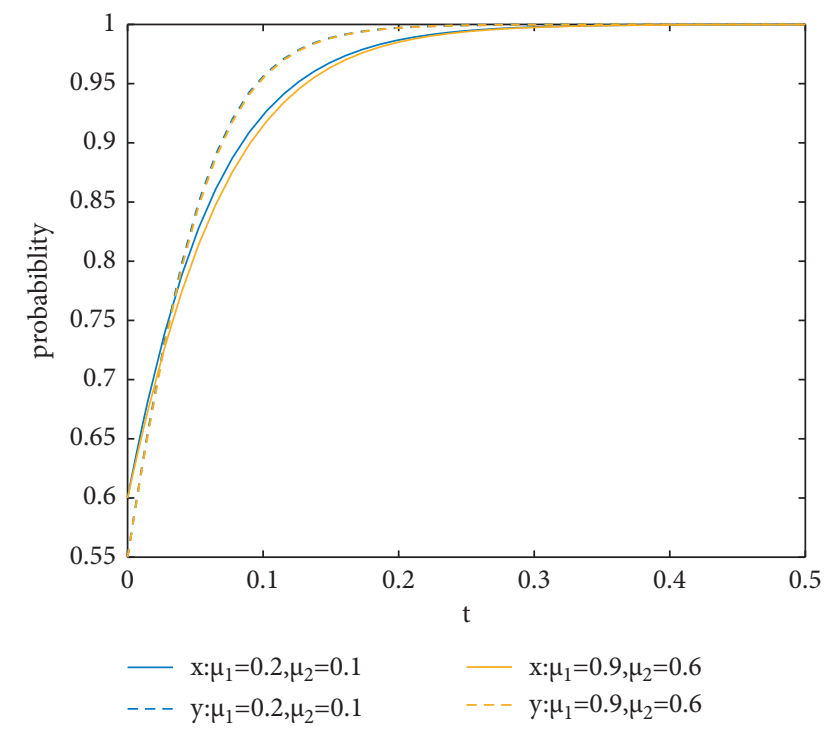

FIgURE 7: The effect of collaborative innovation ability on knowledge hiding.

the possibility of knowledge hiding increases. However, when the stock of knowledge increases to a certain extent, the saddle point becomes larger, the possibility of knowledge hiding decreases, the stable equilibrium point finally converges to $A(0,0)$, and the possibility of both members choosing the strategy of nonknowledge hiding increases. When the knowledge stock of organizational members is low, they lack the self-efficacy of knowledge sharing. To avoid ridicule, they choose knowledge hiding habitually. With the increase in knowledge stock, the possibility of knowledge hiding decreases, but knowledge hiding behavior is not eliminated. Only when the knowledge stock reaches a

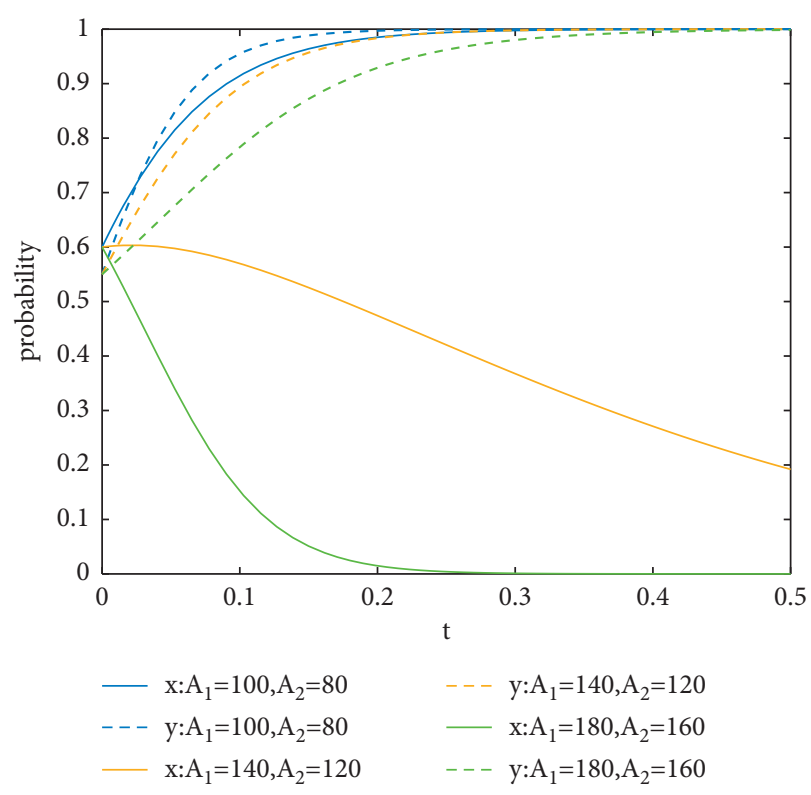

Figure 8: The effect of knowledge stock on knowledge hiding.

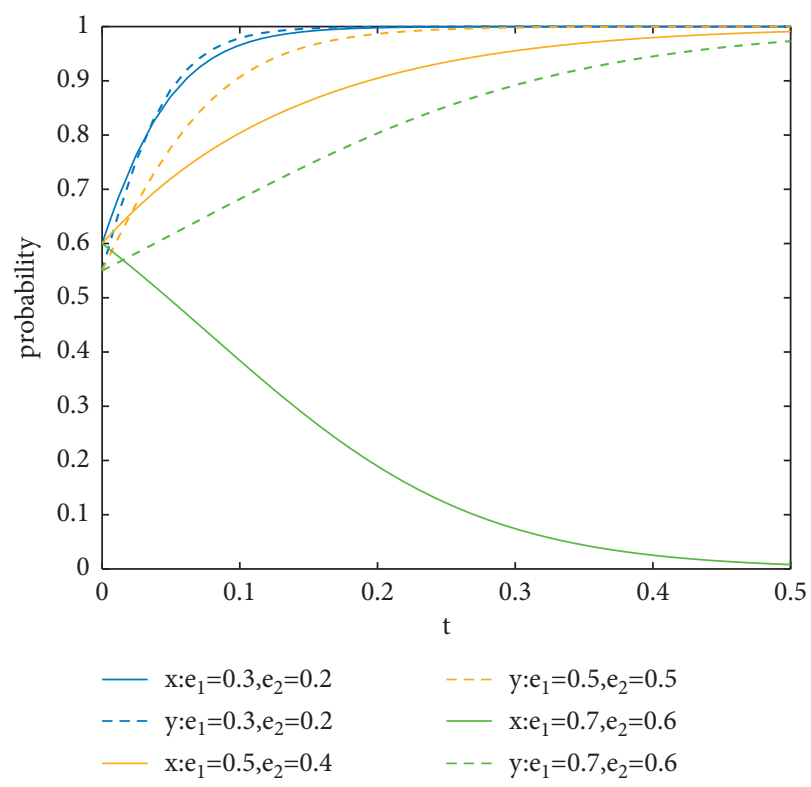

FIgURE 9: The effect of incentive level for cooperation on knowledge hiding.

certain level and members perceive that there is sufficient knowledge sharing self-efficacy in the organization will they give up knowledge hiding and choose adequate knowledge sharing and knowledge exchange.

5.5. Effect of Incentive Level for Cooperation. Cooperation is beneficial to the improvement of the overall efficiency and realization of organizational goals. How to make the best use of the incentive mechanism to promote cooperation among organizational members is important. As shown in Figure 9, when the increase in the incentive level of cooperation and 
keeping the value of other parameters unchanged, when the degree of incentive is low, the saddle point decreases, and the possibility of knowledge hiding increases. However, when the incentive increases to a certain extent, the saddle point becomes larger, the possibility of knowledge hiding decreases, the stable equilibrium point finally converges to $A(0,0)$, and the possibility of both members choosing the strategy of nonknowledge hiding increases. When the incentive from the organization and the team is at a low level, the probability of knowledge hiding decreases with the increase of incentive, but it cannot prevent the occurrence of knowledge hiding behavior. Only when the incentive reaches a certain level can the members be inspired to give up knowledge hiding and carry out full knowledge sharing and exchange. In conclusion, sufficient extrinsic motivation has a good effect on knowledge hiding. It comes from management fairness, positive leadership style, scientific job design, and other related factors.

\section{Conclusion and Implications}

In this paper, an evolutionary game model is established to explore the crucial factors influencing the knowledge hiding strategies of organizational members. By analyzing crucial factors such as knowledge leakage risk, knowledge absorption and transformation ability, collaborative innovation ability, knowledge stock, and the incentive of the organization and team, several meaningful findings are presented. The higher the risk of knowledge leakage, the greater the probability of knowledge hiding. Knowledge absorption and transformation ability is positively correlated with knowledge hiding behavior. When collaborative innovation ability is poor, the possibility of organizational members hiding significantly increases, and with the improvement of collaborative innovation ability, the possibility of knowledge hiding slowly decreases. When the knowledge stock is low, organizational members will choose knowledge hiding habitually, and as the knowledge stock increases, the possibility of knowledge hiding decreases to 0 . Only when the incentive reaches a certain level can the members be inspired to give up knowledge hiding. Existing studies mainly focus on evolutionary game analysis from the perspective of knowledge sharing among organizational members but lack evolutionary game analysis from the perspective of knowledge hiding. This paper builds an evolutionary game model of organizational member knowledge hiding, analyzes the dynamic changes and stabilization strategies of organizational member knowledge hiding from a multidimensional perspective with different parameter variables, and uses numerical simulation to reflect the influence of dynamic changes in different parameters on knowledge hiding strategies. On the one hand, the research results can provide a reference for the research methods of knowledge hiding and expand knowledge management theory. On the other hand, they can provide a useful practical reference for managers to prevent the occurrence of knowledge hiding of organizational members in advance and break the vicious circle of knowledge hiding afterwards.
These results provide important implications for organizations and valuable guidance for managers. The organization should combine strict management with humanistic care and strive to create a tolerant and relaxed environment in the organization. Through education and training, the knowledge stock of organizational members can be increased, the self-efficacy of knowledge sharing among organizational members can be improved, and collaborative innovation among members can be promoted. Through quality development training, the awareness of mutual help among members can be improved to establish a prosocial organizational structure, strengthen the trust among members, and reduce the risk of knowledge leakage. Managers should establish a learning organization, create a good organizational learning atmosphere, and improve the internal knowledge absorption capacity and knowledge transfer efficiency of organizational members. Organizations also need to play an important role in knowledge leadership to shape the prosocial influence of knowledge orientation. In daily work, team knowledge leaders should emphasize the prosocial significance of knowledge contribution, help and support team members in a timely manner, and guide them to transform their professional abilities into an influence to weaken the tendency of knowledge hiding. In addition, a reward system, including material and nonmaterial rewards, should be established to reward organizational members who actively share knowledge so that they can feel the benefits of knowledge sharing. In particular, clear and powerful incentives are praise and encouragement for knowledge workers in organizations to share knowledge, which can make them more sensitive to perceived incentives, thus reducing the probability of knowledge hiding [61].

6.1. Limitations and Directions for Future Research. The higher the risk of knowledge leakage organizational members are aware of, the greater the probability of knowledge hiding. However, individual characteristics are different, and their perceptions and evaluations of risk are different. Therefore, it will be very interesting to study the relationships between individual characteristics and knowledge hiding in the future. Knowledge absorption and transformation ability is positively correlated with knowledge hiding behavior, and when collaborative innovation ability is poor, the possibility of organizational members engaging in knowledge hiding significantly increases. With the improvement of collaborative innovation ability, the possibility of knowledge hiding slowly decreases. According to Fong et al. [33], knowledge hiding reduces the creativity of organizations, mainly because the transfer and absorption of knowledge cannot be fully realized. They suggest solving this problem through task-dependent job design and educational training. However, Gagné et al. [34] proposed that taskdependent work design can lead to knowledge hiding; therefore, how to strengthen knowledge transfer among organizational members needs further study. The knowledge stock is positively correlated with the knowledge hiding behavior of organizational members. Bogilović et al. [51] affirmed that a high level of cultural intelligence could buffer 
the occurrence of knowledge hiding behavior. The different research results may be related to the individual characteristics and organizational culture of organizational members, which will require further discussion in the future. When the organization incentives are sufficient, the possibility of members' knowledge hiding behaviors will be significantly decreased. Therefore, it is worth exploring a scientific and comprehensive organizational incentive method to intervene in the knowledge hiding of organizational members.

This study offers some findings, and there are several other factors influencing knowledge hiding strategies that have been ignored. For example, we can try to construct specific incentive and cost parameter variables and analyze the factors that influence the knowledge hiding of organizational members. In the future, the evolutionary games of different types of knowledge hiding strategies among members of different types of organizations can be studied, and a hybrid strategy evolutionary game model can be built to further define the evasive knowledge hiding behaviors of organizational members.

\section{Data Availability}

The data used to support the findings of this study are included within the article.

\section{Conflicts of Interest}

The authors declare no conflicts of interest.

\section{Acknowledgments}

This work was supported by the Education Department Project of Sichuan (Grant nos. XXTYCY2021D11 and DM201721).

\section{References}

[1] H. W. Liu and C. Feng, "An empirical study on the relationship between technology M\&A model and innovation performance based on knowledge-based view," Science \& Technology Progress and Policy, vol. 32, no. 16, pp. 69-75, 2015.

[2] A. W. King and C. P. Zeithaml, "Measuring organizational knowledge: a conceptual and methodological framework," Strategic Management Journal, vol. 24, no. 8, pp. 763-772, 2003.

[3] B. Van den Hooff and M. Huysman, "Managing knowledge sharing: emergent and engineering approaches," Information \& Management, vol. 46, no. 1, pp. 1-8, 2009.

[4] C. Hamilton and S. P. Philbin, "Knowledge based view of university tech transfer-A systematic literature review and meta-analysis," Administrative Sciences, vol. 10, no. 3, p. 62, 2020.

[5] L. Liu, G. Chen, and X. Niu, "Game analysis of the knowledge sharing mechanism for the supply chain collaborative innovation," Journal of Industrial Engineering and Management, vol. 8, no. 1, pp. 152-169, 2015.

[6] M. Lee, "Knowledge management and innovation management: best practices in knowledge sharing and knowledge value chain," International Journal of Innovation \& Learning, vol. 19, no. 2, pp. 206-226, 2016.

[7] C. Zheng, "Evolutionary game analysis of knowledge sharing in low-carbon innovation network," Complexity, vol. 2021, pp. 1-11, 2021.

[8] S. Yeşil, A. Koska, and T. Büyükbeşe, "Knowledge sharing process, innovation capability and innovation performance: an empirical study," Second International Conference on Leadership, Technology and Innovation Management, vol. 75, pp. 217-225, 2013.

[9] R. Hartono and M. L. Sheng, "Knowledge sharing and firm performance: the role of social networking site and innovation capability," Technology Analysis \& Strategic Management, vol. 28, no. 3, pp. 335-347, 2016.

[10] S. K. Singh, "Territoriality, task performance, and workplace deviance: empirical evidence on role of knowledge hiding," Journal of Business Research, vol. 97, pp. 10-19, 2019.

[11] L. Fonseca, A. Amaral, and J. Oliveira, "Quality 4.0: the EFQM 2020 model and industry 4.0 relationships and implications," Sustainability, vol. 13, no. 6, p. 3107, 2021.

[12] R. Jiang and Y. He, "An empirical study on workplace knowledge hidden behavior," Science and Technology Management Research, vol. 33, no. 20, pp. 128-132, 2013.

[13] X. Zhang and Y. Xi, "The influence mechanism of ethical leadership on employee knowledge hiding," Soft Science, vol. 30, no. 10, pp. 96-99, 2016.

[14] C. E. Connelly, D. Zweig, J. Webster, and J. P. Trougakos, "Knowledge hiding in organizations," Journal of Organizational Behavior, no. 33, pp. 64-88, 2012.

[15] Y. Wang, M. S. Han, D. Xiang, and D. P. Hampson, "The double-edged effects of perceived knowledge hiding: empirical evidence from the sales context," Journal of Knowledge Management, vol. 23, no. 2, pp. 279-296, 2019.

[16] H. Peng, "Counterproductive work behavior among Chinese knowledge workers," International Journal of Selection and Assessment, vol. 20, no. 2, pp. 119-138, 2012.

[17] H. Li and L. Lv, "Influence of prevention focus and motivational climateon knowledge hiding in enterprises," Science Research Management, vol. 40, no. 4, pp. 245-255, 2019.

[18] H. Zhao and Q. Xia, "Interpersonal distrust, negative affect and knowledge hiding behaviors," Science Research Management, vol. 40, no. 8, pp. 284-292, 2019.

[19] H. Zhao and W. Liu, "Visualizing the landscape and trend ofknowledge hiding research," Foreign Economics \& Management, vol. 42, no. 5, pp. 121-138, 2020.

[20] R. S. Lazarus, "Cognition and motivation in emotion," American Psychologist, vol. 46, no. 4, pp. 352-367, 1991.

[21] R. S. Lazarus, "Progress on a cognitive-motivational-relational theory of emotion," American Psychologist, vol. 46, no. 8, pp. 819-834, 1991.

[22] L. Yuan, L. Zhang, and Y. Tu, "The curvilinear relationship between humble leadership and employee knowledge hiding," Soft Science, vol. 32, no. 11, pp. 86-88, 2018.

[23] J. K. Jha and B. Varkkey, "Are you a cistern or a channel? Exploring factors triggering knowledge-hiding behavior at the workplace: evidence from the Indian R\&D professionals," Journal of Knowledge Management, no. 22, pp. 824-849, 2018.

[24] Y. Yuan, L. Yang, X. Cheng, and J. Wei, "What is bullying hiding? Exploring antecedents and potential dimension of knowledge hiding," Journal of Knowledge Management, vol. 25, no. 5, pp. 1146-1169, 2020.

[25] C. E. Connelly and D. Zweig, "How perpetrators and targets construe knowledge hiding in organizations," European 
Journal of Work and Organizational Psychology, vol. 24, no. 3, pp. 479-489, 2015.

[26] C. E. Connelly and E. K. Kelloway, Predictors of employee's perceptions of knowledge sharing cultures, vol. 24, no. 5, pp. 294-301, 2003.

[27] A. M. Abubakar, E. Behravesh, H. Rezapouraghdam, and S. B. Yildiz, "Applying artificial intelligence technique to predict knowledge hiding behavior," International Journal of Information Management, vol. 49, pp. 45-57, 2019.

[28] U. Ghani, T. Teo, Y. Li et al., "Tit for tat: abusive supervision and knowledge hiding-the role of psychological contract breach and psychological ownership," International Journal of Environmental Research \& Public Health, vol. 17, no. 4, p. 1240, 2020.

[29] Z. Jiang, X. Hu, Z. Wang, and X. Jiang, "Knowledge hiding as a barrier to thriving: the mediating role of psychological safety and moderating role of organizational cynicism," Journal of Organizational Behavior, vol. 40, no. 7, pp. 800-818, 2019.

[30] Y. Zhu, T. Chen, M. Wang, Y. Jin, and Y. Wang, "Rivals or allies," How performance - prove goal orientation influences knowledge hiding, vol. 40, no. 7, pp. 849-868, 2019.

[31] Y. Su and Y. Yu, "Spatial agglomeration of new energy industries on the performance of regional pollution control through spatial econometric analysis," The Science of the Total Environment, vol. 704, p. 135261, 2020.

[32] M. Černe, T. Hernaus, A. Dysvik, and M. Škerlavaj, “The role of multilevel synergistic interplay among team mastery climate, knowledge hiding, and job characteristics in stimulating innovative work behavior," Human Resource Management Journal, vol. 27, no. 2, pp. 281-299, 2017.

[33] P. S. W. Fong, C. Men, J. Luo, and R. Jia, "Knowledge hiding and team creativity: the contingent role of task interdependence," Management Decision, vol. 56, no. 2, 2018.

[34] M. Gagné, A. W. Tian, C. Soo, B. Zhang, K. S. B. Ho, and K. Hosszu, "Different motivations for knowledge sharing and hiding: the role of motivating work design," Journal of Organizational Behavior, vol. 40, no. 7, pp. 783-799, 2019.

[35] H. Zhao, Q. Xia, P. He, G. Sheard, and P. Wan, "Workplace ostracism and knowledge hiding in service organizations," International Journal of Hospitality Management, vol. 77, pp. 290-291, 2016.

[36] Y. Su, X. Jiang, and Z. Lin, "Simulation and Relationship Strength: Characteristics of Knowledge Flows Among Subjects in a Regional Innovation System," Science, Technology and Society, 2021.

[37] F. Offergelt, M. Spörrle, K. Moser, and J. D. Shaw, "Leader signaled knowledge hiding: effects on employees' job attitudes and empowerment," Journal of Organizational Behavior, vol. 40, no. 7, pp. 819-833, 2019.

[38] F. Wei and Y. Ma, "Supervisor's territorial behavior and employee s knowledge hiding behavior: a moderated mediation model," Industrial Engimeering and Management, vol. 23, no. 4, pp. 179-185, 2018.

[39] X. Zhai and Y. Shu, "Investigating the knowledge hiding behavior in online learning community and its driving mechanism: a perspective from professional commitment and transformative leadership," The Journal of Distance Education, vol. 37, no. 5, pp. 85-94, 2019.

[40] C. Men, P. S. W. Fong, W. Huo, J. Zhong, R. Jia, and J. Luo, "Ethical leadership and knowledge hiding: a moderated mediation model of psychological safety and mastery climate," Journal of Business Ethics, vol. 166, no. 3, pp. 461-472, 2018.
[41] P. He, X. Wang, and Z. Li, "Effect of abusive supervision on subordinates' knowledge hiding behavior-the roles of moral disengagement and supervisor-subordinate guanxi," East China Economic Management, vol. 32, no. 8, pp. 144-151, 2018.

[42] H. Zhao, W. Liu, J. Li, and X. Yu, "Leader-member exchange, organizational identification, and knowledge hiding: the moderating role of relative leader-member exchange," Journal of Organizational Behavior, vol. 40, no. 7, pp. 834848, 2019.

[43] K. Babič, M. Černe, C. E. Connelly, A. Dysvik, and M. Škerlavaj, "Are we in this together? Knowledge hiding in teams, collective prosocial motivation and leader-member exchange," Journal of Knowledge Management, vol. 23, no. 8, pp. 1502-1522, 2019.

[44] H. Guo, L. Zhang, S. Hong, and S. Huang, "The effect of prosocial sensemaking mechanisms on knowledge withholding intension: a perspective of knowledge leadership," Chinese Journal of Management, vol. 17, no. 1, pp. 111-120, 2020.

[45] T. Hernaus, M. Cerne, C. Connelly, N. P. Vokic, and M. Škerlavaj, "Evasive knowledge hiding in academia: when competitive individuals are asked to collaborate," Journal of Knowledge Management, vol. 23, no. 4, pp. 597-618, 2019.

[46] C. Xiong, V. Change, V. Scuotto, Y. Shi, and N. Paoloni, "The social-psychological approach in understanding knowledge hiding within international $\mathrm{R} \& \mathrm{D}$ teams: an inductive analysis," Journal of Business Research, vol. 128, pp. 799-811, 2021.

[47] A. S. Butt and A. B. Ahmad, "Are there any antecedents of topdown knowledge hiding in firms? Evidence from the United Arab Emirates," Journal of Knowledge Management, vol. 23, no. 8, pp. 1605-1627, 2019.

[48] C. E. Connelly, D. P. Ford, O. Turel, B. Gallupe, and D. Zweig, "I'm busy (and competitive)!' Antecedents of knowledge sharing under pressure," Knowledge Management Research and Practice, vol. 12, no. 1, pp. 74-85, 2014.

[49] M. Škerlavaj, C. E. Connelly, M. Cerne, and A. Dysvik, "Tell me if you can: time pressure, prosocial motivation, perspective taking, and knowledge hiding," Journal of Knowledge Management, vol. 22, no. 7, pp. 1489-1509, 2018.

[50] Z. Geofroy and M. M. Evans, "Are emotionally intelligent employees less likely to hide their knowledge?" Knowledge and Process Management, vol. 24, no. 2, pp. 81-95, 2017.

[51] S. Bogilović, M. Černe, and M. Škerlavaj, "Hiding behind a mask? Cultural intelligence, knowledge hiding, and individual and team creativity," European Journal of Work and Organizational Psychology, vol. 26, no. 5, pp. 710-723, 2017.

[52] R. B. Rezwan and Y. Takahashi, "The psychology behind knowledge hiding in an organization," Administrative Sciences, vol. 11, no. 2, p. 57, 2021.

[53] L. Lin, J. Shi, and D. Tang, "Knowledge sharing incentive of project teams based on the perspective of knowledge withholding," Science Research Management, vol. 36, no. 5, pp. 162-170, 2015.

[54] A. Serenko and N. Bontis, "Understanding counterproductive knowledge behavior: antecedents and consequences of intraorganizational knowledge hiding," Journal of Knowledge Management, vol. 20, no. 6, pp. 1199-1224, 2016.

[55] O. F. Malik, A. Shahzad, M. M. Raziq, M. M. Khan, S. Yusaf, and A. Khan, "Perceptions of organizational politics, knowledge hiding, and employee creativity: the moderating role of professional commitment," Personality \& Individual Differences, vol. 142, pp. 232-237, 2019. 
[56] F. Jiang, J. Zhang, Q. Yang, and H. Cheng, "Research on the game of knowledge hidden behavior based on prospect theory," Science and Technology Management Research, vol. 40, no. 22, pp. 190-195, 2020.

[57] K. Zhang, Y. Li, and W. Guo, "Research on the influence of intellectual property risk on cooperative behavior in R\&D," Management Review, vol. 23, no. 12, pp. 76-83, 2011.

[58] J. Mi, R. Lin, K. Dong, and P. Song, "An evolutionary game analysis on knowledge sharing among OFDI corporation and local cluster enterprises: based on the perspective of knowledge potential," Management Review, vol. 28, no. 9, pp. 106-120, 2016.

[59] M. Guo, L. Zhang, and Y. Zhu, "The impact of territoriality on employee innovative behavior," Business Management Journal, vol. 42, no. 5, pp. 88-102, 2020.

[60] H. Yao, X. Mao, and H. Jin, "Research on evolutionary game of knowledge sharing in virtual community considering platform influencing factors," Operations Research and Management Science, vol. 29, no. 12, pp. 82-88, 2020.

[61] Y. Gao and B. Sun, "The large-small group-based evolutionary game on knowledge sharing in uncertain environment under the background of telemedicine service," Complexity, vol. 2020, Article ID 9818417, 13 pages, 2020. 